\title{
Overexpression analysis suggests that FON2-LIKE CLE PROTEIN1 is involved in rice leaf development
}

\author{
Yoshihiro Ohmori ${ }^{\dagger}$, Yukiko Yasui and Hiro-Yuki Hirano* \\ Department of Biological Sciences, Graduate School of Science, The University of Tokyo, \\ Bunkyo-ku, Tokyo 113-8654, Japan
}

(Received 16 April 2014, accepted 9 May 2014)

\begin{abstract}
Peptide signaling plays important roles in various developmental processes of plants. Genes encoding CLE proteins, which are processed into CLE signaling peptides, are required for maintenance of the shoot apical meristem and for vascular differentiation. FON2-LIKE CLE PROTEIN1 (FCP1), a member of the CLE gene family, negatively regulates meristem maintenance in both shoot and root apical meristems of rice (Oryza sativa). Here, we examined the role of FCP1 in leaf development. We found that overexpression of FCP1 affects various aspects of leaf development in shoots regenerated from calli, making it difficult to distinguish between the leaf blade and leaf sheath. Differentiation of tissues such as vascular bundle and sclerenchyma was strongly inhibited by $F C P 1$ overexpression. Spatial expression patterns of developmental genes DROOPING LEAF $(D L)$ and OsPINHEAD1 (OsPNH1) were severely affected in the FCP1-overexpressing shoots. Whereas $D L$ was expressed in the central region of leaf primordia in control shoots, $D L$ expression was expanded throughout the leaf primordia of the FCP1-overexpressing shoots in early developmental stages. By contrast, $O s P N H 1$, which is expressed in provascular and developing vascular tissues in normal seedlings, was strongly repressed by FCP1 overexpression. Taken together, our results suggest that $F C P 1$ is involved in the regulation of cell fate determination during leaf development.
\end{abstract}

Key words: cell differentiation, CLE peptide, $F C P 1$, leaf development, rice

Intercellular communication is particularly important for development in plants, because plant cells are fixed by rigid cell walls and do not migrate. Many types of small peptides are involved in communication between cells and between functional domains within tissues and organs to regulate developmental processes such as stem cell maintenance, vascular differentiation, and stomatal development. In Arabidopsis thaliana, stem cell maintenance in the shoot apical meristem is negatively regulated by a CLAVATA3 (CLV3) CLE peptide, which acts through receptor complexes such as CLV1, CLV2/CRN, and RPK2 (reviewed in Aichinger et al., 2012; Ha et al., 2010; Miyawaki et al., 2013). Mutation in CLV3 leads to an enlarged meristem consisting of over-proliferated stem cells, due to a failure in the repression of WUSCHEL (WUS) activity, which promotes stem cell identity (Fletcher et al., 1999). Conversely, overexpression of $C L V 3$ results in premature termination of the meristem

\footnotetext{
Edited by Koji Murai

* Corresponding author. E-mail: hyhirano@bs.s.u-tokyo.ac.jp

$\dagger$ Present address: Graduate School of Agriculture and Life Sciences, The University of Tokyo, Yayoi, Bunkyo, Tokyo 1138657, Japan
}

(Brand et al., 2000; Fletcher et al., 1999).

In rice, mutations in the genes FLORAL ORGAN NUMBER1 (FON1) and FON2 give rise to enlargement of the floral meristem, resulting in increases in the numbers of floral organs such as carpels and stamens (Suzaki et al., 2004, 2006). FON1 encodes a CLV1-like LRR receptor kinase, whereas the protein encoded by FON2 contains a CLE domain highly similar to that of Arabidopsis CLV3. Unlike Arabidopsis clv mutants, however, rice fon mutants do not display abnormal phenotypes in the vegetative phase. In addition, overexpression of FON2 does not affect vegetative development, whereas it disturbs inflorescence and flower development probably due to defects in maintenance of the reproductive meristem. Thus, CLV signaling appears to be partially conserved in rice (reviewed in Miyawaki et al., 2013; Pautler et al., 2013).

FON2-LIKE CLE PROTEIN1 (FCP1) encodes a CLE protein closely related to FON2 and plays an important role in the vegetative development of rice (Suzaki et al., 2008; Ohmori et al., 2013). Constitutive overexpression of $F C P 1$ causes premature termination of the meristem in shoots regenerating from calli (Suzaki et al., 2008). Like- 
wise, inducible overexpression of $F C P 1$ results in a failure in meristem maintenance (Ohmori et al., 2013). When FCP1 expression is strongly induced, the undifferentiated cell marker OSH1 and the stem cell marker FON2 are downregulated and leaf initiation is compromised. Conversely, inducible knockdown of $F C P 1$ and $F C P 2$ leads to increased expression of OSH1 and FON2 and expansion of the expression domains of these genes, suggesting that meristem activity is upregulated. Although it is not known whether a WUS ortholog functions similarly in rice, a member of the WUSCHEL-RELATED HOMEO$B O X(W O X)$ gene family, OsWOX4, is involved in meristem maintenance (Ohmori et al., 2013). Inducible knockdown of OsWOX4 results in downregulation of OSH1 and FON2. Consistent with this observation, the expression of $O s W O X 4$ is negatively regulated by $F C P 1$. Thus, the role of $F C P 1$ in meristem maintenance is relatively well understood. In the current work, we have analyzed the function of FCP1 in other developmental processes such as leaf development and shoot regeneration.

We previously found that overexpression of FCP1 (FCP1-OX) results in the regeneration of abnormal shoots from calli (Suzaki et al., 2008). To examine the role of FCP1 in regeneration, we characterized the regeneration process in detail. Calli were induced from the scutellum and used for Agrobacterium-mediated transformation (Hiei et al., 1994). After selection for hygromycin resistance, calli were transferred to regeneration medium. In calli carrying the empty vector, tiny green spots were observed about $10 \mathrm{~d}$ after transfer, and green leaf-like organs were subsequently observed (Fig. 1, A and B). Shoot-like structures and roots were formed after 25 and $30 \mathrm{~d}$ on the regeneration medium, respectively (Fig. 1, C and D). Normal shoots, which were very similar in appearance to those of seedlings, were obtained within $40 \mathrm{~d}$ (Fig. 1E). By contrast, regeneration was delayed in the FCP1-OX calli. Even after $18 \mathrm{~d}$ on the regeneration medium, we observed only green calli that lacked leaf-like structures (Fig. 1, F and G). Many FCP1-OX calli failed to form a shoot-like structure after $30 \mathrm{~d}$ (Fig. 1, $\mathrm{H}$ and I). After $40 \mathrm{~d}$, shoot-like structures with malformed morphology were apparent, but no root regeneration was observed in the FCP1-OX calli (Fig. 1J). At this stage, we often observed abnormal calli that produced several small, leaf-like organs (Fig. 1K). The shoots that did form on FCP1-OX calli did not grow further and eventually died. Thus, FCP1 overexpression disturbed shoot regeneration from callus and resulted in the production of malformed shoots.

To elucidate the effects of $F C P 1$ overexpression, we first made cross-sections of regenerating shoots and observed the inner tissues. Normal leaves were formed in the shoots carrying empty vector, and leaf blades and leaf sheaths were easily distinguished from one another, as in seedlings germinated from seeds (Fig. 2, A and G). The leaf blade contained small cytoplasm-dense cells, whereas the leaf sheath had larger cells that were less stained with toluidine blue. Vascular bundles were differentiated in both the leaf blade and leaf sheath, with large and small vascular bundles distinguishable in the leaf blade (Fig. 2, $\mathrm{A}$ and $\mathrm{H}$ ). In the leaf sheath, sclerenchyma tis-

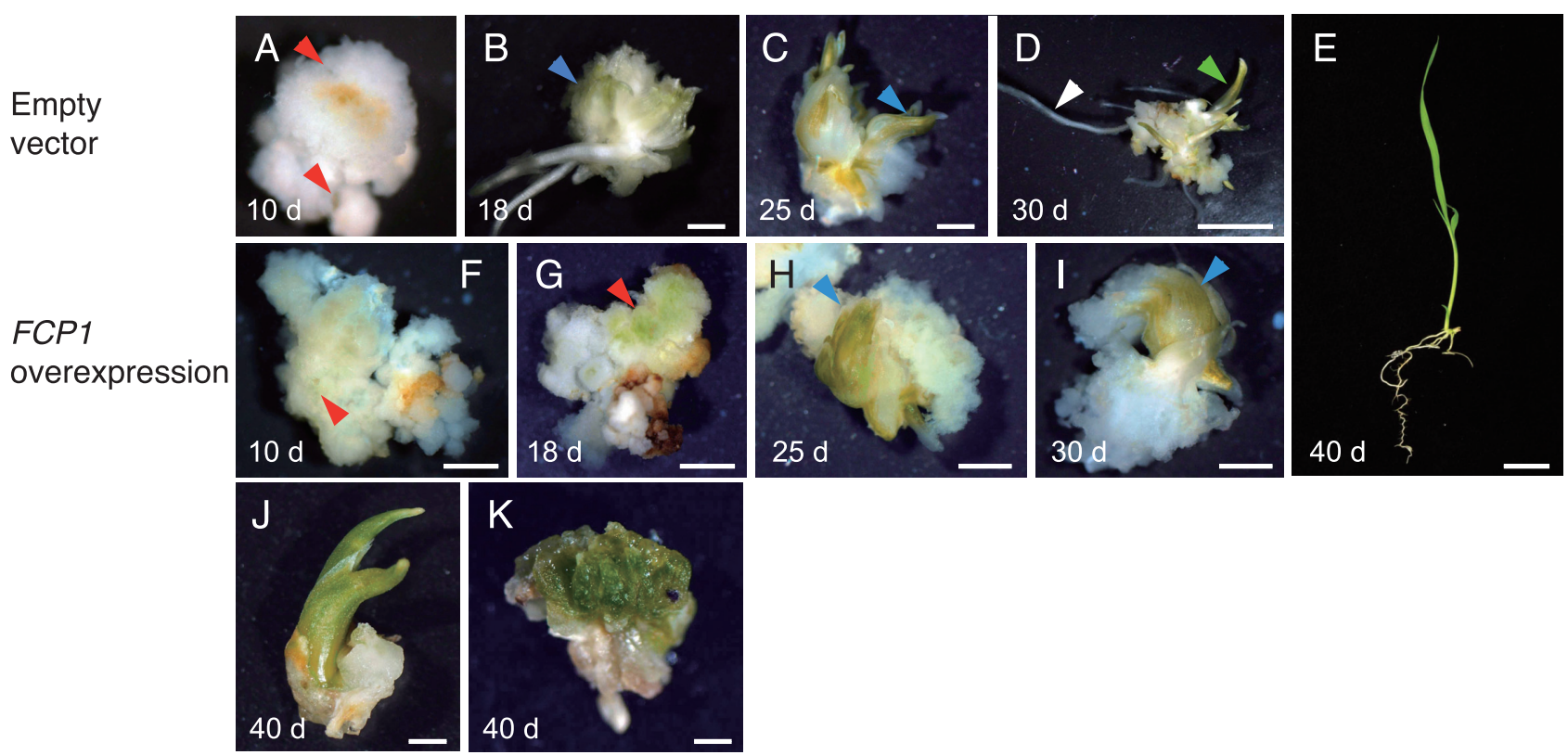

Fig. 1. Shoot regeneration from callus. (A-E) Calli carrying empty vector. (F-K) FCP1-OX calli. The number of days after transfer to the regeneration medium is indicated. Arrowheads indicate the following: red, green spot (A, F, G); blue, leaf-like structure (B, C, H, I); green, shoot (D); white, root (D). Scale bars = $1 \mathrm{~mm}$ in (A-D, F-K), $1 \mathrm{~cm}$ in (E). 
Empty vector

FCP1 overexpression
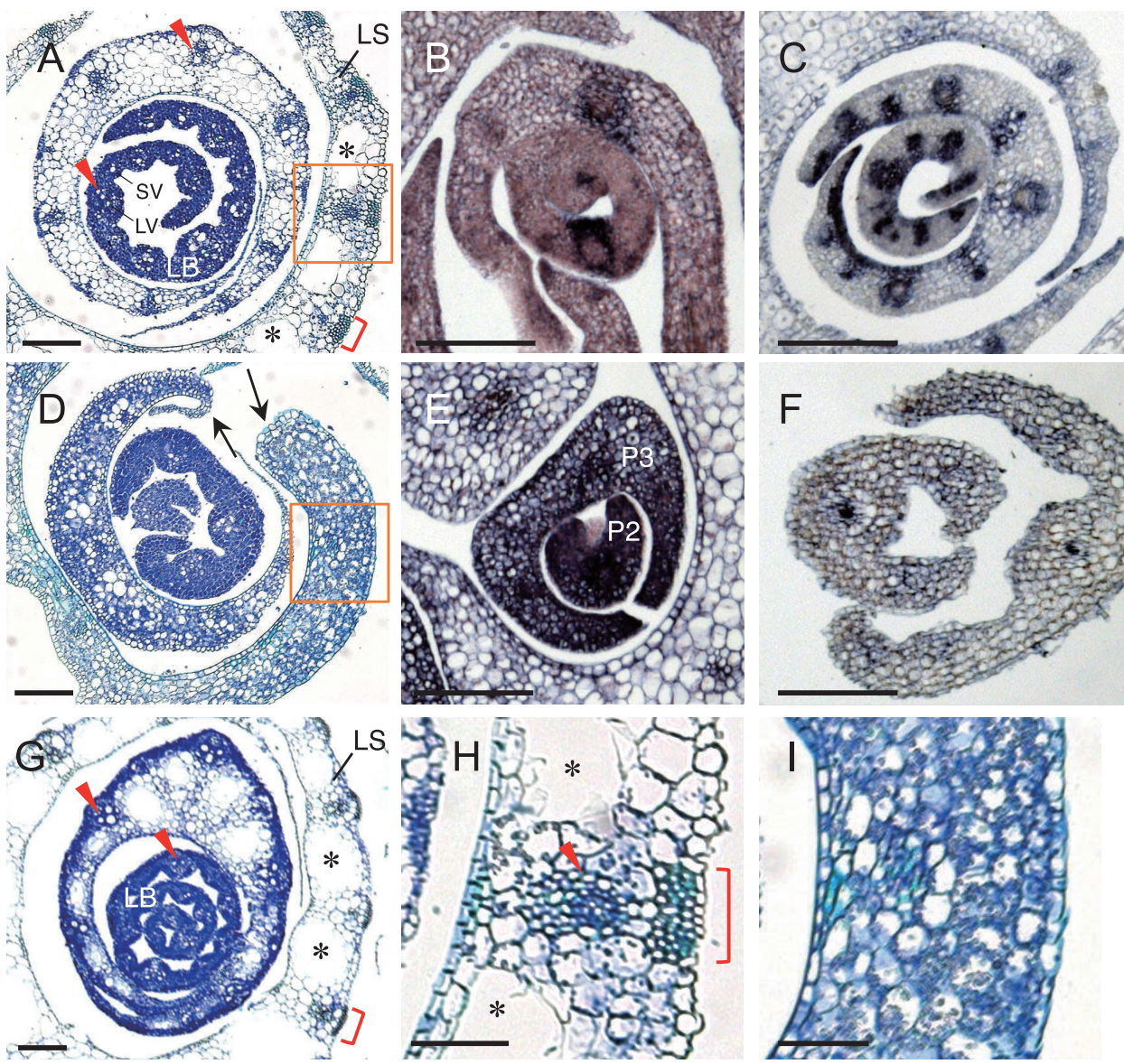

Fig. 2. Effect of FCP1 overexpression on cell differentiation and expression of marker genes. (A-C, H) Regenerating shoots carrying empty vector. (D-F, I) FCP1-OX regenerating shoots. (G) A shoot germinated from a seed. (A, D) Cross-section of the apex of the regenerating shoots. (B, E) In situ hybridization showing spatial distribution of DL transcripts. (C, F) In situ hybridization showing spatial distribution of $\mathrm{OsPNH}$ transcripts. (H, I) Close-up views of the regions indicated with squares in (A) and (D), respectively. (G) Cross-section of the shoot apex of a seedling germinated from a seed. Arrowheads and asterisks indicate vascular bundles and air spaces, respectively. Arrows indicate abnormal margins. Brackets indicate sclerenchyma tissues. LB, leaf blade; LS, leaf sheath; LV, large vascular bundle; SV, small vascular bundle. Scale bars $=100 \mu \mathrm{m}$ in $(\mathrm{A}-\mathrm{G})$, $50 \mu \mathrm{m}$ in $(\mathrm{H}, \mathrm{I})$.

sues adaxial to the vascular bundles were differentiated and air spaces were formed (Fig. 2H). By contrast, it was hard to distinguish between leaf blade and leaf sheath in the FCP1-OX shoots (Fig. 2D). Although vascular-like structures were sometimes observed in inner leaves, their number and tissue patterns were abnormal. In outer leaves, no vascular-like tissues were formed (Fig. 2, D and I). Sclerenchyma and air spaces were also absent (Fig. 2, D and I). In addition, the morphology of the marginal region was abnormal (Fig. 2D). Taken together, these observations showed that cell differentiation was highly compromised in the FCP1-OX shoots.

Next, we examined the expression patterns of two genes, DROOPING LEAF (DL) and OsPINHEAD1 (OsPNH1), that are expressed in leaf primordia (Nishimura et al., 2002; Ohmori et al., 2011; Yamaguchi et al., 2004). DL is required for midrib formation in the leaf and is expressed in the central region of the leaf primordium
(Ohmori et al., 2011; Yamaguchi et al., 2004). In shoots carrying empty vector, $D L$ transcripts were localized to the central region (Fig. 2B), as in the wild type seedlings. By contrast, $D L$ transcripts were observed in the FCP1$O X$ shoots throughout the leaf primordia at earlier developmental stages (P2 and P3), but were absent in the leaf primordia at subsequent stages (Fig. 2E). Thus, the spatial and temporal expression pattern of $D L$ was deregulated in the FCP1-OX shoots.

OsPNH1 is responsible for leaf development and meristem maintenance (Nishimura et al., 2002). In the empty-vector control shoots, OsPNH1 was expressed in the presumptive region of vascular differentiation and the adaxial region of the leaf primordia (Fig. 2C), as previously described (Nishimura et al., 2002). By contrast, OsPNH1 expression in the FCP1-OX shoots was not detected in the leaf primordia except for weak expression in a narrow domain of the leaf center (Fig. 2F). These 
data suggest that overexpression of FCP1 strongly represses $O s P N H 1$ expression in the regenerating shoot.

We previously showed that $F C P 1$ is expressed in the leaf primordia in addition to the shoot apical meristem in wild-type shoot, suggesting that FCP1 functions in leaf development (Suzaki et al., 2008). Our current results from overexpression analysis suggest that $F C P 1$ plays a role in determining cell fate during leaf development. In control regenerating shoots, as in seedlings germinated from seeds, cells differentiate to produce the vascular bundle, sclerenchyma, and marginal tissues. By contrast, cellular differentiation was strongly inhibited in the FCP1-OX shoots. The cells in the FCP1-OX leaf primordia appeared largely homogeneous at a stage when the control had formed leaf sheath cells (Fig. 2, D and I). In addition, air space formation, a process involving programmed cell death in wild-type leaf sheaths (Inada et al., 2002), was absent in the FCP1-OX shoots.

Expression of developmental genes was also altered by FCP1 overexpression, which caused $D L$ and $O s P N H 1$ to be up- and downregulated throughout young leaf primordia, respectively (Fig. 2, E and F). DL promotes cell proliferation in the central region of the leaf primordia in normal seedlings (Ohmori et al., 2011; Yamaguchi et al., 2004). The leaf primordia were thicker in the FCP1-OX shoots than in the control (Fig. 2, A and D), which may result from enhanced cell proliferation caused by ectopic $D L$ expression in the $F C P 1-O X$ shoots. In the wild-type shoot, FCP1 is expressed throughout the leaf primordia from the P1 to P3 stage, whereas both $D L$ and $O s P N H 1$ are expressed in specific and distinct regions from $\mathrm{P} 1$ to P4 (Nishimura et al., 2002; Ohmori et al., 2011; Yamaguchi et al., 2004). This spatiotemporally overlapping expression pattern between $F C P 1$ and $D L / O s P N H 1$ is consistent with the idea that $F C P 1$ is likely to be involved in the regulation of both $D L$ and $O s P N H 1$ in wild type. We conclude that an appropriate level of FCP1 expression appears to be required for normal leaf development through the function of key developmental factors regulating cell differentiation.

Differentiation of vascular tissues was compromised in FCP1-OX shoots. OsPNH1 was expressed in the presumptive regions of vascular differentiation and developing vascular bundles in the control, whereas this $O s P N H 1$ expression was strongly repressed by FCP1 overexpression (Fig. 2, C and F). It has been reported that antisense suppression of $O s P N H 1$ results in partial failure of vascular differentiation (Nishimura et al., 2002). Therefore, defects in vascular development in the FCP1overexpressing shoots may be caused by the downregulation of OsPNH1. Alternatively, downregulation of OsWOX4 may be associated with a failure of vascular development in the FCP1-OX shoots. In Arabidopsis, WOX4 plays a crucial role in the maintenance of vascular stem cells (Hirakawa et al., 2010). In rice, we have shown that $O s W O X 4$ is expressed in the provascular and developing vascular tissues, suggesting that OsWOX4 is involved in vascular development as well as meristem maintenance (Ohmori et al., 2013). In addition, OsWOX4 is negatively regulated by FCP1 (Ohmori et al., 2013). It is therefore also plausible that the failure in vascular development in the FCP1-OX shoots is due to downregulation of $\mathrm{OsWOX} 4$. These two hypotheses are not mutually exclusive.

Progress in shoot regeneration was delayed in FCP1$O X$ shoots. This suggests that cell cycle progression or cell proliferation is partially compromised by FCP1 overexpression. This delay may also be related to the disturbance of cell differentiation.

Together, our results suggest that FCP1 is involved in the regulation of cell differentiation in leaf development, likely affecting the expression of many genes. This highlights the importance of understanding FCP1 signaling and its downstream genetic networks in the leaf.

We thank E. Oki for technical assistance. This research was supported in part by Grants-in-Aid for Scientific Research from MEXT (23248001, 25113008 to H.-Y. H.).

\section{REFERENCES}

Aichinger, E., Kornet, N., Friedrich, T., and Laux, T. (2012) Plant stem cell niches. Annu. Rev. Plant Biol. 63, 615-636.

Brand, U., Fletcher, J. C., Hobe, M., Meyerowitz, E. M., and Simon, R. (2000) Dependence of stem cell fate in Arabidopsis on a feedback loop regulated by $C L V 3$ activity. Science 289, 617-619.

Fletcher, J. C., Brand, U., Running, M. P., Simon, R., and Meyerowitz, E. M. (1999) Signaling of cell fate decisions by CLAVATA3 in Arabidopsis shoot meristems. Science $\mathbf{2 8 3}$, 1911-1914.

Ha, C. M., Jun, J. H., and Fletcher, J. C. (2010) Shoot apical meristem form and function. Curr. Top. Dev. Biol. 91, 103140.

Hiei, Y., Ohta, S., Komari, T., and Kumashiro, T. (1994) Efficient transformation of rice (Oryza sativa L.) mediated by Agrobacterium and sequence analysis of the boundaries of the T-DNA. Plant J. 6, 271-282.

Hirakawa, Y., Kondo, Y., and Fukuda, H. (2010) TDIF peptide signaling regulates vascular stem cell proliferation via the WOX4 homeobox gene in Arabidopsis. Plant Cell 22, 26182629 .

Inada, N., Sakai, A., Kuroiwa, H., and Kuroiwa, T. (2002) Threedimensional progression of programmed death in the rice coleoptile. Int. Rev. Cytol. 218, 221-258.

Miyawaki, K., Tabata, R., and Sawa, S. (2013) Evolutionarily conserved CLE peptide signaling in plant development, symbiosis, and parasitism. Curr. Opin. Plant Biol. 16, 598606.

Nishimura, A., Ito, M., Kamiya, N., Sato, Y., and Matsuoka, M. (2002) OsPNH1 regulates leaf development and maintenance of the shoot apical meristem in rice. Plant J. 30, 189-201.

Ohmori, Y., Toriba, T., Nakamura, H., Ichikawa, H., and Hirano, H.-Y. (2011) Temporal and spatial regulation of DROOPING $L E A F$ gene expression that promotes midrib formation in 
rice. Plant J. 65, 77-86.

Ohmori, Y., Tanaka, W., Kojima, M., Sakakibara, H., and Hirano, H. Y. (2013) WUSCHEL-RELATED HOMEOBOX4 is involved in meristem maintenance and is negatively regulated by the CLE gene FCP1 in rice. Plant Cell 25, 229241.

Pautler, M., Tanaka, W., Hirano, H.-Y., and Jackson, D. (2013) Grass meristem I - Shoot apical meristem maintenance, axillary meristem determinacy and the floral transition. Plant Cell Physiol. 54, 302-312.

Suzaki, T., Sato, M., Ashikari, M., Miyoshi, M., Nagato, Y., and Hirano, H.-Y. (2004) The gene FLORAL ORGAN NUMBER1 regulates floral meristem size in rice and encodes a leucinerich repeat receptor kinase orthologous to Arabidopsis
CLAVATA1. Development 131, 5649-5657.

Suzaki, T., Toriba, T., Fujimoto, M., Tsutsumi, N., Kitano, H., and Hirano, H.-Y. (2006) Conservation and diversification of meristem maintenance mechanism in Oryza sativa: function of the FLORAL ORGAN NUMBER2 gene. Plant Cell Physiol. 47, 1591-1602.

Suzaki, T., Yoshida, A., and Hirano, H.-Y. (2008) Functional diversification of CLAVATA3-related CLE proteins in meristem maintenance in rice. Plant Cell 20, 2049-2058.

Yamaguchi, T., Nagasawa, N., Kawasaki, S., Matsuoka, M., Nagato, Y., and Hirano, H.-Y. (2004) The YABBY gene DROOPING LEAF regulates carpel specification and midrib development in Oryza sativa. Plant Cell 16, 500-509. 